IUCM93-010

\title{
Addition Spectra of Quantum Dots in Strong Magnetic Fields
}

\author{
S.-R. Eric Yangt: and A.H. MacDonald \\ Indiana University, Department of Physics, Bloomington IN 47405 \\ M.D. Johnson \\ Department of Physics, University of Central Florida, Orlando FL 32816-2385
}

(May 4, 2018)

\begin{abstract}
We consider the magnetic field dependence of the chemical potential for parabolically confined quantum dots in a strong magnetic field. Approximate expressions based on the notion that the size of a dot is determined by a competition between confinement and interaction energies are shown to be consistent with exact diagonalization studies for small quantum dots. Fine structure is present in the magnetic field dependence which cannot be explained without a full many-body description and is associated with groundstate level crossings as a function of confinement strength or Zeeman interaction strength. Some of this fine structure is associated with precursors of the bulk incompressible states responsible for the fractional quantum Hall effect.
\end{abstract}


Advances in nanofabrication technology have made it possible to realize artificial systems in which electrons are confined to a small area within a two dimensional electron gas. Interest in these 'quantum dot' systems [1] has been enhanced as a result of recently developed techniques [2,3] which probe them spectroscopically. The quantity which is measured [4] in these experiments is the magnetic field dependence of the 'addition spectrum', i.e., the energy to add one electron to a dot. This is given by $\mu_{N} \equiv E_{N}^{0}-E_{N-1}^{0}$ where $E_{N}^{0}$ is the ground state energy of an $N$-electron dot. Addition spectrum measurements have generally been interpreted in terms of 'constant interaction' models in which electronelectron interactions within a quantum dot are accounted for by including a charging energy which is characterized by a fixed self-capacitance; or, when this fails, by using Hartree or Hartree-Fock approximations. However, especially at strong magnetic fields, quantum dots can have strongly correlated [5,6] ground states, some of which are precursors of the bulk incompressible states responsible for the fractional quantum Hall effect. In this regime a complete interpretation of addition spectra measurements requires an exact treatment of the Coulombic electron-electron interactions.

In this Letter we report on numerical exact diagonalization calculations of the addition spectrum for quantum dots in a strong magnetic field. We find that the addition spectrum has a surprisingly rich magnetic field dependence, showing a large number of sharp features superimposed on a smooth background. The smooth background can be accounted for using a simple Hartree approximation. The sharp features are associated with energy-level crossings at fixed $N$, often between strongly correlated states. The role of the spin degree of freedom is non-trivial and is not in general consistent with expectations of exchangeenhanced spin-splitting based on the Hartree-Fock approximation. The constant interaction model fails qualitatively for strong magnetic fields.

We consider a system of electrons in two dimensions (2D) which are confined by a parabolic external potential [7], $V(r)=m \Omega^{2} r^{2} / 2$. We confine our attention here to the strong magnetic field limit [8], $\Omega / \omega_{c} \geq 1 .\left(\omega_{c} \equiv e B_{\perp} / m c\right.$ is the cyclotron frequency and $B_{\perp}$ is the component of the magnetic field perpendicular to the $2 \mathrm{D}$ electron gas.) In this limit 
[1] the symmetric gauge single-particle eigenstates are conveniently classified by a Landau level index $n$ and an angular momentum index $m=-n, \ldots, \infty$, and we can confine our attention to $n=0$. The single-particle orbitals in the lowest Landau level have energies $\varepsilon_{m}=\hbar \omega_{c} / 2+\gamma(m+1)$, where $\gamma=m \Omega^{2} \ell^{2}=\hbar \Omega^{2} / \omega_{c}$ and $\ell^{2} \equiv \hbar c / e B_{\perp} . \quad\left(\left\langle m\left|r^{2}\right| m\right\rangle=\right.$ $2 \ell^{2}(m+1)$.) The quantized kinetic energy of the lowest Landau level is a constant which hereafter we absorb into the zero of energy. We use as the unit of energy the interaction energy $e^{2} / \epsilon \ell$. Then the many-electron energies are determined by two dimensionless numbers characterizing the ratio of the confinement and Zeeman energies to the interaction energy; respectively, $\tilde{\gamma} \equiv \gamma /\left(e^{2} / \epsilon \ell\right)$ and $\tilde{g} \equiv g \mu B /\left(e^{2} / \epsilon \ell\right)$. Note that we explicitly include the possibility of tilted fields since we believe that tilted-field experiments will prove to be very valuable. As discussed below we have evaluated the ground state energy over a wide range of values for these two parameters.

The Hamiltonian in our system is invariant under spatial rotations about an axis perpendicular to the 2D plane and passing through the center of the quantum dot, and also under rotations in spin space about the magnetic field direction $(\hat{\alpha})$. It follows that both the total angular momentum $M_{z}$ and $S_{\alpha} \equiv \vec{S} \cdot \hat{\alpha}$ are good quantum numbers. It is straightforward to choose a representation for the many-body Hamiltonian which is diagonal in these two operators and block diagonal for the Hamiltonian. Eigenenergies may be expressed as a sum of interaction and single-particle contributions,

$$
E_{i}\left(N, M_{z}, S_{\alpha}\right)=U_{i}\left(N, M_{z}, S_{\alpha}\right)+\gamma\left(N+M_{z}\right)-g \mu_{B} B S_{\alpha} .
$$

Here $i$ labels a state within a $\left(M_{z}, S_{\alpha}\right)$ subspace, and $U_{i}\left(N, M_{z}, S_{\alpha}\right) \propto e^{2} / \epsilon \ell$ is determined by exactly diagonalizing the electron-electron interaction term in the Hamiltonian within this subspace [9]. In our study we have used a Lanczos algorithm to determine only the minimum interaction energy within each subspace, $U_{0}\left(N, M_{z}, S_{\alpha}\right)$. For $N=2,3,4,5,6$ we have considered all possible values of $S_{\alpha}$, while for $N=7,8$ we have considered only fully spin polarized states with $S_{\alpha}=N / 2$. In each case we have considered all values of $M_{z}$ from the minimum value consistent with the Pauli exclusion principle (see below) to $M_{z}=$ 
$3 N(N-1) / 2$, which is large enough to accommodate an $m=3$ Laughlin droplet [10,11]. For given values of $\tilde{\gamma}$ and $\tilde{g}$ the subspace containing the ground state is determined by minimizing $U_{0}\left(N, M_{z}, S_{\alpha}\right)+\tilde{\gamma}\left(N+M_{z}\right)-\tilde{g} S_{\alpha}$ over all values of $M_{z}$ and $S_{\alpha}$ for which calculations have been performed. This procedure results in a surprisingly rich phase diagram for a quantum dot.

Results for $N=5$ and $N=6$ are shown in Fig. (1) and Fig. (22). Regions in the phase diagram are labeled by by $\left(M_{z}, 2 S_{\alpha}\right)$, the quantum numbers of the state with lowest energy. Along the boundary lines in these phase diagrams ground state level crossings occur; the slope of a line is given by $\left(S_{\alpha}-S_{\alpha}^{\prime}\right) /\left(M_{z}-M_{z}^{\prime}\right)$ and the intercept by $\left(U_{0}\left(N, M_{z}^{\prime}, S_{\alpha}^{\prime}\right)-\right.$ $\left.U_{0}\left(N, M_{z}, S_{\alpha}\right)\right) /\left(M_{z}-M_{z}^{\prime}\right)$. It follows from the spin-rotational invariance of the electronelectron interaction term in the Hamiltonian that states may be labeled by a total spin quantum number $S$ and by $S_{\alpha}=-S, \cdots, S$. In each spin multiplet the only ground state candidate for any non-zero $\tilde{g}$ is the state which is polarized along the field, i.e. $S_{\alpha}=S$. Thus the $S_{\alpha}$ values in these figures give the total spin quantum numbers of the corresponding states.

We discuss these rather complicated phase diagrams, beginning with $\tilde{g}$ and $\tilde{\gamma}$ relatively large, on the upper right-hand side of the figures. For $N=5$ only the $(4,1),(6,3)$, and $(10,5)$ regions in the phase diagram correspond to the single Slater determinant ground states which would be obtained in the Hartree-Fock approximation. The occupation numbers for these states are given by $(\bullet \bullet \bullet ; \bullet \bullet \circ),(\bullet \bullet \bullet \bullet \circ ; \bullet)$, and $(\bullet \bullet \bullet \bullet \bullet \circ ; \circ)$, respectively. $[$ An occupied(unoccupied) single-particle state is represented by a full(empty) circle. Circles left(right) of the semicolon represent spin up(down) states. The angular momentum $m$ of a single-particle state increases from left to right.] Similarly, for $N=6$ the $(6,0),(7,2),(10,4)$, and $(15,6)$ regions have single Slater determinant ground states with occupation numbers given by $(\bullet \bullet \bullet ; \bullet \bullet \bullet),(\bullet \bullet \bullet \bullet ; \bullet \bullet \circ),(\bullet \bullet \bullet \bullet \bullet ; \bullet)$, and $(\bullet \bullet \bullet \bullet \bullet \circ ; \circ)$ respectively. The $(4,1)$ state for $N=5$ and the $(6,0)$ state for $N=6$ minimize the confinement energy and are ground states at all values of $\tilde{\gamma}$ in the absence of electron-electron and Zeeman interactions. These states are the precursors of the Landau level filling factor $\nu=2$ states 
for bulk systems.

As the confinement strength $\tilde{\gamma}$ weakens, interactions favor less compact (larger total angular momentum) electron dots $\left[U_{0}\left(N, M_{z}+1, S_{\alpha}\right) \leq U_{0}\left(N, M, S_{\alpha}\right)\right]$. For these dot sizes the expansion is first accomplished, except at small $\tilde{g}$, by forming the most compact states consistent with increasing spin polarization until complete spin polarization is reached. For large $\tilde{g}$, states with large spin quantum numbers are favored; eventually, for very large $\tilde{g}$, only states with $S=N / 2$ occur. The tendency toward complete spin polarization is what simplifies the phase diagram at larger values of $\tilde{g}$. At small $\tilde{g}$, as the confinement $\tilde{\gamma}$ weakens the dot expands by introducing holes [11,12] into the interior of the dot. As these holes begin to correlate the Hartree-Fock approximation begins to fail. One consequence is that interactions often favor states which are not completely spin-polarized. At weaker confinement the ground states are linear combinations of many Slater determinants. Many of the states which occur can be identified as precursors of the bulk incompressible states responsible for the fractional quantum Hall effect. For example for $N=5$ the $(30,5)$ region corresponds to the $\nu=\frac{1}{3}$ state while for $N=6$ the $(36,0)$ and $(45,6)$ phase regions correspond to the $\nu=\frac{2}{5}$ spin-singlet state and $\nu=\frac{1}{3}$ spin-polarized states.

States with larger values of $M_{z}$ occur and the phase diagram becomes richer as $\tilde{\gamma}$ decreases. The upper panels in Fig. (11) and Fig. (2) show the small $\tilde{g}$, small $\tilde{\gamma}$ regions of the phase diagrams on an expanded scale. The dashed line shows the path taken through these phase diagrams for a GaAs sample with $\hbar \Omega=2 \mathrm{meV}$ as a function of a perpendicular magnetic field. (For GaAs $\tilde{\gamma} \sim 0.131(\hbar \Omega[\mathrm{meV}])^{2} /\left(B_{\perp}[\text { Tesla }]\right)^{3 / 2}$ and $\tilde{g} \sim 0.0059 B[$ Tesla $] /\left(\mathrm{B}_{\perp}[\text { Tesla }]\right)^{1 / 2}$.) Regions of the phase diagram to the right of this line could be explored experimentally by using tilted magnetic fields.

Some qualitative features of these results can be understood using a simple argument which considers the competition between the Hartree energy and the confinement energy of a quantum dot. We assume that in the ground state electrons occupy the $N_{\phi}$ smallest$m$ orbitals with approximately equal probability, leading to a charge distribution which 
is approximately that of a uniform disk of radius [13] $R=\ell \sqrt{2 N_{\phi}}$. (For such a state $M_{z} \sim N N_{\phi} / 2$. The maximum value of $N / N_{\phi}$ allowed by the Pauli exclusion principle is 1 for spin-polarized states and 2 for unpolarized states.) For all but the smallest dots the two largest contributions to the total energy will be the Hartree energy,

$$
E_{H} \sim \frac{8 e^{2} N^{2}}{3 \pi \epsilon R}=\frac{e^{2}}{\epsilon \ell} \frac{4 \sqrt{2}}{3 \pi} \frac{N^{2}}{N_{\phi}^{1 / 2}}
$$

and the confinement energy,

$$
E_{C}=\gamma\left(M_{z}+N\right) \sim \gamma N N_{\phi} / 2
$$

Corrections due to exchange and correlations (which reduce the interaction energy below $\left.E_{H}\right)$ give a contribution proportional to $N^{1}$ for large $N$ are relatively less important for large dots. The confinement energy favors compact dots with small values of $N_{\phi}$ while the interaction energy favors expanded dots. For a given value of $\tilde{\gamma}$ and $N$ the optimum dot size can be determined by minimizing $E_{H}+E_{C}$ with respect to $N_{\phi}$. This gives

$$
\begin{gathered}
\frac{N_{\phi}}{N}=\left(\frac{4 \sqrt{2}}{3 \pi \tilde{\gamma} N^{1 / 2}}\right)^{2 / 3}, \\
E_{H}+E_{C}=\frac{3}{2}\left[\left(e^{2} / \epsilon \ell\right)^{2} \gamma(4 \sqrt{2} / 3 \pi)^{2}\right]^{1 / 3} N^{5 / 3},
\end{gathered}
$$

and

$$
\mu_{N} \sim \frac{5}{2}\left[\left(e^{2} / \epsilon \ell\right)^{2} \gamma(4 \sqrt{2} / 3 \pi)^{2}\right]^{1 / 3} N^{2 / 3}
$$

Note that in this approximation the energy and $\mu_{N}$ are independent of magnetic field. This result differs qualitatively from the constant interaction model where $\mu_{N}$ would be the sum of an interaction term proportional to $N$ and a single-particle term. The difference here is due to the fact that the size of the dot is not fixed but is determined by a competition of interaction and single-particle terms. Comparing with Fig. (11) and Fig. (2) we see that the values of the ground state angular momenta are reasonably estimated [1] by Eq. (4) (using $M_{z} \sim N N_{\phi} / 2$ ) even for $N=5$ and $N=6$. (Overestimates are expected since correlations 
will reduce the interaction energy cost of making the dots smaller.) The above Hartree argument predicts $N_{\phi}$ or $M_{z}$ in the ground state. In a Hartree-Fock generalization of this argument the exchange energy would stabilize the state with the largest spin polarization allowed for a given $M_{z}$ by the Pauli exclusion principle. Indeed the most compact fully spin-polarized state $\left(M_{z}=N(N-1) / 2 ; S_{\alpha}=N / 2\right)$, which is the precursor of the bulk $\nu=1$ state, has a large range of stability in the phase diagrams of Fig. (1) and Fig. (2). However, as seen most clearly in the upper panels, many states with smaller values of $S_{\alpha}$ occur at larger $M_{z}$ where full spin polarization is allowed. This is in direct contradiction with Hartree-Fock theory and is a result of correlations.

Fig. (3) shows [14 the magnetic field dependence of $\mu_{6}$ for a GaAs sample with $\hbar \Omega=2 \mathrm{meV}$. (The inset shows results for $N=2,3,4,5,6$ on a wider energy scale.) The approximately $N^{2 / 3}$ dependence of $\mu_{N}$ at fixed field and the weak magnetic-field dependence are explained by Eq. (6). Similarly, in approximate agreement with Eq. (四), the angular momentum difference between the $N=5$ ground state and the $N=6$ ground state increases from 5 to 15 in going from the left- to right-hand sides of the curve. However, the finer features apparent in the plot of $\mu_{6}$ can be understood only by accounting for the possibility of strong correlations in the quantum dot and cannot be explained with Hartree-Fock or similar approximations. The apparently smooth curve for $N=6$ in the inset can be seen to have a large number of cusps due to ground state level crossings for either $N=5$ or $N=6$ quantum dots. At a ground state level crossing $d E_{0} / d B$ must decrease. It follows that ground state level crossings in the $N-1$ and $N$ particle systems lead respectively to positive and negative jump discontinuities in $d \mu_{N} / d B$ as seen in Fig. (3). Note that unlike the prediction of an independent-particle approximation [2,3], upward and downward pointing cusps do not in general alternate. At the left-hand side $(B \sim 2.5$ Tesla $)$ of this figure both the $N=5$ and $N=6$ dots are in the $\left(M_{z}=N(N-1) / 2 ; S_{\alpha}=N / 2\right)$ maximum-density spin-polarized single Slater determinant states, while at the right-hand side $(B \sim 6$ Tesla $)$ both $N=5$ and $N=6$ dots are in $\left(M_{z}=3 N(N-1) / 2 ; S_{\alpha}=N / 2\right)$ states. These states are the precursors of the bulk $\nu=1$ and $\nu=1 / 3$ incompressible states and the incompressibility 
is reflected [9] in the relative large regions of stability in the phase diagrams. The resulting 'plateaus' in the addition spectrum should be among the most visible features experimentally. Precursors of a Landau level filling factor $\nu$ state will occur for $N / N_{\phi}=\nu$; it follows from Eq. (4) that for GaAs we can expect associated features in the addition spectrum to occur for $B[$ Tesla $] \sim 0.363(\hbar \Omega[\mathrm{meV}])^{4 / 3} N^{1 / 3} / \nu$. Features identified with $\nu=2$ in the recent experiments of Ashoori et al. [3] seem to follow this $N^{1 / 3}$ law rather well. We believe that the unidentified experimental features which appear at approximately twice this field are associated with precursors of the $\nu=1$ incompressible state which is stabilized primarily be electron-electron interactions. We predict that features associated with precursors of fractional incompressible states will appear at stronger fields and also, less visibly, at intermediate fields.

This work was supported by the National Science Foundation under grant DMR-9113911 and by the UCF Division of Sponsored Research. AHM acknowledges helpful conversations with Ray Ashoori, Mark Kastner, Horst Stormer and Karen Tevosyan. 


\section{REFERENCES}

* Permanent address: IMS, NRC, Ottawa K1A 0R6, Canada

[1] For recent reviews see U. Merkt, Advances in Solid State Physics, 30, 77 (1990); Tapash Chakraborty, Comments on Condensed Matter Physics 16, 35 (1992); M.A. Kastner, Rev. Mod. Phys. 64, 849 (1992).

[2] P.L. McEuen, E.B. Foxman, U. Meirav, M.A. Kastner, Y. Meir, Ned S. Wingreen, and S.J. Wind, Phys. Rev. Lett. 66, 1926 (1991); P.L. McEuen, E.B. Foxman, Jari Kinaret, U. Meirav, M.A. Kastner, Ned S. Wingreen, and S.J. Wind, Phys. Rev. B. 45, 11419 (1992); E.B. Foxman, P. L. McEuen, U. Meirav, Ned S. Wingreen, Yigal Meir, Paul A. Belk, N. R. Belk, and M.A. Kastner, and S.J. Wind, Phys. Rev. B 47, 10020 (1993).

[3] R.C. Ashoori, H.L. Stormer, J.S. Weiner, L.N. Pfeiffer, S.J. Pearton, K.W. Baldwin, and K.W. West, Phys. Rev. Lett. 68, 3088 (1992); R.C. Ashoori, H.L. Stormer, J.S. Weiner, L.N. Pfeiffer, K.W. Baldwin, and K.W. West, submitted to Phys. Rev. Lett. (1993).

[4] Y. Meir, N.S. Wingreen, and P.A. Lee, Phys. Rev. Lett. 66, 3048 (1991); C.W.J. Beenakker, Phys. Rev. B 44, 1646 (1991).

[5] Correlation effects in two-electron dots have been related to the addition spectrum by Ashoori et al. Two-electron dots have been studied numerically by M. Wagner, U. Merkt, and A.V. Chaplik, Phys. Rev. B 45, 1951 (1992) and by Daniela Pfannkuche, Vidar Gudmundsson, and Peter A. Maksym, Phys. Rev. B 47, 2244 (1993).

[6] P.A. Maksym and T. Chakraborty, Phys. Rev. Lett. 65, 108 (1990); J.M. Kinaret Yigal Meir, Ned S. Wingreen, Patrick Lee, and Xiao-Gang Wen, Phys. Rev. B 45, 9489 (1992); N.F. Johnson and M.C. Payne, Phys. Rev. Lett. 67, 1157 (1991).

[7] For the small $N$ quantum dots considered here the use of a parabolic confinement potential model is well justified. For quantum dots defined by gates the potential from external 
charges will change in a geometry-dependent way as the quantum dot is charged. These changes can often be accounted for in terms of phenomenological capacitances.

[8] As discussed further below the strong magnetic field limit applies for increasingly large $N$ dots as the magnetic field strength increases.

[9] Technical details will be described in a planned longer publication.

[10] Sami Mitra and A.H. MacDonald, Phys.Rev. B, (to appear) 1993.

[11] A.H. MacDonald and M.D. Johnson, Phys. Rev. Lett. 70, 3107 (1993).

[12] A.H. MacDonald, S.-R. Eric Yang, and M.D. Johnson, Australian Journal of Physics, to appear (1993).

[13] The assumption of an approximately uniform charge density is a good one except for very large dots when the ground state of the dot is related to an incompressible state of the two-dimensional electron gas. For very large dots the classical semielliptical density profile will be approached. For a discussion of the classical limit see, for example, S. Nazin, K. Tevosyan, and V. Shikin, Surf. Sci. 263, 351 (1992) and work cited therein.

[14] A code which generates addition spectra from the many-electron eigenenergy data sets is available from the authors for use in interpreting experimental results. 


\section{FIGURES}

FIG. 1. Phase diagram for a $N=5$ parabolically confined quantum dot. Regions in the phase diagram are labeled by the $M_{z}$ and $N_{\uparrow}-N_{\downarrow}$ values of the ground state. $\left(S_{\alpha}=\left(N_{\uparrow}-N_{\downarrow}\right) / 2\right.$.) The upper panel shows the rich behavior at weak confinement which is related to the physics of the fractional quantum Hall effect. The dashed line shows the path in the phase diagram followed by GaAs sample with $\hbar \Omega=2 \mathrm{meV}$ and a perpendicular magnetic field between $B=2.5$ Tesla and $B=7$ Tesla.

FIG. 2. Phase diagram for a $N=6$ parabolically confined quantum dot. Regions in the phase diagram are labeled as in Fig. (1).

FIG. 3. Magnetic field dependence of the $N=6$ addition spectrum for a parabolically confined quantum dot with $\hbar \Omega=2 \mathrm{meV}$. The curve has a cusp whenever there is a ground state level crossing for either the $N=5$ or $N=6$ dot. Curve segments between two upward tick marks are labeled with the ground state quantum numbers $\left(M_{z}, 2 S_{z}\right)$ of the $N=6$ dot. Segments between downward tick marks are labeled with the quantum numbers of the $N=5$ dot. The paths followed through the phase diagrams for this model are indicated by the dashed lines in Fig. (1) and Fig. (2). The inset shows results for $N=2,3,4,5,6$ on a wider energy scale. The dashed lines in the inset result from the Coulomb blockade model, with a phenomenological self-capacitance obtained by a fit to the exact numerical results. 